\title{
Fréchet differentiability in statistical inference for time series
}

\author{
Tadeusz Bednarski
}

Accepted: 31 May 2010 / Published online: 24 June 2010

(C) The Author(s) 2010. This article is published with open access at Springerlink.com

\begin{abstract}
It is shown how the method of Fréchet differentiability can simplify the asymptotic derivations in an important range of robust inferential problems for stationary and related time series models. The uniform root- $n$ consistency of the empirical distribution function for the Cramer von Mises norm under a weak mixing condition is indicated. Various regularity conditions naturally implemented and leading to the differentiability are discussed. A simulation study supplementing the theoretical discussion is included.
\end{abstract}

Keywords Time series $\cdot$ Robust inference $\cdot$ Differentiability

Mathematics Subject Classification (2000) $\quad 62 \mathrm{~F} 35 \cdot 62 \mathrm{E} 20$

\section{Introduction}

The literature on statistical inference for stationary time series shows complexity of distributional problems on both technical and assumptive side, especially in the context of robust inference, compare for instance Mancini et al. (2005) and Muler et al. (2009). Testing the unit root hypotheses, in spite of many successful efforts, even in the nonrobust context, is still a "difficult" problem. Moreover, the stationarity assumption, so fundamental in empirical economic studies, has there an approximative meaning. Phillips (2004) indicates that the degree of nonstationarity in economic data often differs significantly from one variable to another (interest rates, inflation, income). If we add to this frequent institutional and legislative changes, political turnovers,

T. Bednarski $(\varangle)$

Institute of Economic Sciences, Wroclaw University, ul. Uniwersytecka 22/26,

50-145 Wroclaw, Poland

e-mail: t.bednarski@prawo.uni.wroc.pl 
international conflicts, that "change the rules of the game" in economies, then it becomes quite clear that the notion of stationarity may apply only to very local in time and space economic processes. Considering the above one may have an uneasy feeling over the statistical inference for the stationary time series in the context of economic applications.

There are, of course, sophisticated robust methods and asymptotic methods designed for specific stationary time series. However a general inferential method, flexible to possible departures from the stationarity assumption and at the same time leading to "simple" asymptotic derivations would certainly be of help. It is the aim of this study is to show that the method of Fréchet differentiability of von Mises functionals can be applied to statistical inference for stationary and related time series models in a way that unifies and simplifies the asymptotic derivations in an important range of problems.

Here, we shall adopt the following definition: a statistical functional $T$ is called Fréchet differentiable at a model distribution $F$ with respect to a norm $\|$.$\| if we have$

$$
T(G)-T(F)=L(G-F)+o(\|G-F\|)
$$

where $L$ is a bounded linear functional (operator) defined on the linear space spanned by the differences of distributions $G-F$.

To apply the above relationship to statistical inference we need a convenient representation of the functional and a statistically meaningful norm. If it is

$$
\int \psi_{F} d(G-F)=L(G-F)
$$

for some function $\psi_{F}$ and $\sqrt{n}\left\|F_{n}-G\right\|$ stays bounded in probability uniformly in $G$, where $F_{n}$ is the empirical distribution based on a sample $X_{1}, \ldots, X_{n}$ then

$$
\sqrt{n}\left(T\left(F_{n}\right)-\theta_{0}\right)=\frac{1}{\sqrt{n}} \sum_{i} \psi_{F}\left(X_{i}\right)+o_{P}(1)
$$

where $\theta_{0}$ is the true parameter value, gives relatively simple, mathematically rigorous and a flexible way to determine the limiting behavior of the estimator $T\left(F_{n}\right)$. In robustness theory $\psi_{F}$ is commonly called influence function, since in more intuitive terms $\psi_{F}(x)$ measures the influence of an outlier with mass at $x$ (Hampel et al. 1986). The differentiability implies that $\psi_{F}$ is bounded.

The applicability of the differentiability notion depends then, on one hand, on distributional properties of $\frac{1}{\sqrt{n}} \sum_{i} \psi_{F}\left(X_{i}\right)$ and, on the other hand, on uniform boundedness in probability of $\sqrt{n}\left\|F_{n}-G\right\|$. When the sample $X_{1}, X_{2}, \ldots, X_{n}$, is iid then the Dvoretzky et al. (1956) inequality, for the empirical distribution functions, leads to uniform root- $n$ consistency of $F_{n}$ in the Kolmogorov Smirnov distance. In the general dependent variable case however, the inequality holds under very strong uniform $\phi$-mixing assumptions (Rio 2000), which seems to narrow applicability of the Kolmogorov Smirnov norm there. However, it turns out that application of the Cramer 
von Mises distance between distribution functions leads to root- $n$ consistency of the empirical distribution function in the case of dependent identically distributed random variables in a much simpler way.

Since the functional $T$ must depend on the empirical distribution function, the methods developed here to facilitate asymptotic studies are meant for situations where statistical inference can be based on structural features of estimable finite dimensional distributions. To be more specific consider a series $X_{t}=\rho X_{t-1}+e_{t}$ where $e_{t}$ is a sequence of centered iid random variables. Inference about the parameter $\rho$ can be carried out using the distribution of $\left(X_{t}, X_{t-1}\right)$, independent of time in strictly stationary case. The two-dimensional distribution can be estimated via the empirical distribution function of the sample consisting of pairs of consecutive variables of the process. This simple way of inference, naturally expandable to ARIMA processes, in fact frequently used in practice, is of course less efficient than inference based on precise knowledge of the process distribution. However, on the other hand, it is less dependent on the distributional assumptions and therefore more flexible and robust.

In the following section a uniform consistency of the empirical distribution functions for the Cramer von Mises norm is shown for dependent variables satisfying some $\alpha$-mixing conditions. Section 3 justifies merits of Fréchet differentiability in terms of regularity of statistical parametric models. Section 4 discusses the local uniform differentiability in the case of dependent observations. Section 5 describes some practical merits of the method in the context of unit root testing via simulation experiments.

\section{Root- $n$ consistency of empirical distribution function for the Cramer-von Mises norm}

The objective here is to study conditions under which the sequence of random variables $\sqrt{n}\left\|F_{n}-F\right\|$, where $F_{n}$ is the empirical distribution function based on a sample consisting of possibly dependent random variables with distribution $F$, is bounded in probability (uniformly in $F$ ). The asymptotic behavior of $\sqrt{n}\left\|F_{n}-F\right\|$ depends of course on the norm choice. Though the Kolmogorov-Smirnov norm works very well in the iid case it seems hardly expandable to practically important sequences of dependent variables in the context of time series. The following result shows utility of the Cramer-von Mises ( $\mathrm{CvM})$ distance, another good candidate in the context of robust inference.

We define the CvM norm here as

$$
\left\|F_{1}-F\right\|^{2}=\int\left(F_{1}-F\right)^{2} d Q
$$

where $Q$ is a probability measure. A good choice for $Q$ could be either a selected model distribution or a uniform distribution over a bounded region of anticipated observed values.

Suppose $X_{1}, X_{2}, \ldots$ is a sequence of random variables, each with distribution $F$ satisfying the following mixing condition 


$$
\sup _{i \in N, t \in R} \sum_{j=0}^{\infty}\left|P\left(X_{j}<t \wedge X_{i}<t\right)-P\left(X_{j}<t\right) P\left(X_{i}<t\right)\right|<K<\infty
$$

Theorem 2.1 Under the condition (1) the random variables $\sqrt{n}\left\|F_{n}-F\right\|$ are bounded in probability, uniformly in $F$.

Proof By Chebyshev's inequality it is sufficient to properly bound the expected value of $n\left\|F_{n}-F\right\|^{2}$. Note that since

$$
n E\left\|F_{n}-F\right\|^{2}=\lim _{m \rightarrow \infty} \sum_{k=2}^{m}\left(Q\left(t_{k}\right)-Q\left(t_{k-1}\right)\right) E\left(1 / n \sum_{i=1}^{n} I_{X_{i} \leq t_{k}}-F\left(t_{k}\right)\right)^{2}
$$

where $t_{1}<t_{2}<\cdots<t_{n}$ form appropriate partitions of $R$; $t_{1}=-\infty, t_{n}=+\infty$, we have, in the iid case,

$$
\begin{aligned}
n E\left\|F_{n}-F\right\|^{2} & =\lim _{m \rightarrow \infty} \sum_{k=2}^{m}\left(Q\left(t_{k}\right)-Q\left(t_{k-1}\right)\right)\left(\frac{n-1}{n} F^{2}\left(t_{k}\right)+\frac{1}{n} F\left(t_{k}\right)-F^{2}\left(t_{k}\right)\right) \\
& =\lim _{m \rightarrow \infty} \sum_{k=1}^{m}\left(Q\left(t_{k}\right)-Q\left(t_{k-1}\right)\right) F\left(t_{k}\right)\left(1-F\left(t_{k}\right)\right) / n \\
& =\frac{1}{n} \int F(t)(1-F(t)) d Q
\end{aligned}
$$

In the case of dependent variables satisfying (1) the following simple majorization holds

$$
\begin{aligned}
\lim _{m \rightarrow \infty} \sum_{k=2}^{m} Q\left(\left(t_{k}\right)-Q\left(t_{k-1}\right)\right) E\left(1 / n \sum_{i=1}^{n} I_{X_{i} \leq t_{k}}-F\left(t_{k}\right)\right)^{2} \\
=\lim _{m \rightarrow \infty} \sum_{k=2}^{m}\left(Q\left(t_{k}\right)-Q\left(t_{k-1}\right)\right)\left[\frac{n-1}{n} F^{2}\left(t_{k}\right)+\frac{1}{n} F\left(t_{k}\right)\right. \\
\left.\quad+\frac{1}{n^{2}} \sum_{i=1, j=1, i \neq j}^{n}\left(F_{i, j}\left(t_{k}, t_{k}\right)-F^{2}\left(t_{k}\right)\right)-F^{2}\left(t_{k}\right)\right] \\
=\frac{1}{n} \int F(t)(1-F(t)) d Q \\
\quad+\lim _{m \rightarrow \infty} \sum_{k=2}^{m}\left(Q\left(t_{k}\right)-Q\left(t_{k-1}\right)\right)\left[\frac{1}{n^{2}} \sum_{i=1, j=1, i \neq j}^{n}\left(F_{i, j}\left(t_{k}, t_{k}\right)-F^{2}\left(t_{k}\right)\right)\right] \\
\leq \frac{1}{n} \int F(t)(1-F(t)) d Q+\frac{K}{n}
\end{aligned}
$$


where $F_{i, j}\left(t_{k}, t_{k}\right)=P\left(X_{i}<t_{k}, X_{j}<t_{k}\right)$. Therefore we can conclude that

$$
P\left(n \int\left(\frac{1}{n} \sum_{i=1}^{n} I_{x_{i} \leq t}-F(t)\right)^{2} d Q>M\right) \leq \frac{\int F(t)(1-F(t)) d Q+K}{M}
$$

holds uniformly in $F$.

There is no difficulty in extending the above argumentation to multivariate $F$ if we apply the Lebesgue-Stieltjes integral. Notice also that to conclude the uniform root- $n$ consistency of the Cramer von Mises norm we need much weaker assumptions than the standard $\alpha$-mixing condition used in time series.

\section{Model regularity and Fréchet differentiability with respect to the Cramer-von Mises norm, the iid case}

The goal of this section is to show a close relationship between regularity of a statistical model, differentiability of statistical functionals and existence of a linear locally uniformly valid expansion of the estimator.

It is well known that model regularity plays an important role in reliable parametric inference. It leads to nice asymptotic properties of MLE estimators and it ensures fixed $1 / \sqrt{n}$ convergence rate of efficient estimates for iid samples. Moreover, locally, the distributions of the sample are in a sense regularly spaced-they neither coincide nor orthogonalize, as the sample size increases. To be more specific suppose $\mathcal{P}=\left\{P_{\theta}\right\}_{\theta \in R^{k}}$ is a parametric model.

The model $\mathcal{P}$ is called regular at $\theta$ if it is $L^{2}$ differentiable at $P_{\theta}$, that is there exists a random variable $\Lambda_{\theta} \in L_{k}^{2}\left(P_{\theta}\right)$ such that

$$
\int\left(\sqrt{p_{\theta+t}}-1-\frac{1}{2} t^{\prime} \Lambda_{\theta}\right)^{2} d P_{\theta}=o\left(|t|^{2}\right)
$$

where $p_{\theta+t}=d P_{\theta+t} / d P_{\theta}$.

Largely simplifying our explanation we can say that locally at $P_{\theta}$ our original model's densities $d P_{\theta+t} / d P_{\theta}$ are closely approximated by another parametric model, so called tangent model, indexed by $t$ and given by the $d P_{\theta}$ densities equal to $1+t^{\prime} \Lambda_{\theta}$. The tangent model is as regular as it can be since the relationship between its probability measures and the parameters is linear and smooth.

When we refer to a Fisher consistent statistical functional then the parametrization at the model is "given" by the functional itself; $T\left(F_{\theta}\right)=\theta$-we prefer to use the the distribution functions $F_{\theta}$ instead of corresponding probability measures $P_{\theta}$ since the accuracy of expansion is given in terms of differences of distribution functions. However in the context of robust inference we expect this parametrization to be naturally expendable to a fully nonparametric neighborhood of $P_{\theta}$. We would also like, as in the parametric case, this parametrization to be locally close to a smooth linear parametrization 


$$
T(G)=T(F)+L(G-F)+o(\|G-F\|)
$$

with $L$ a linear functional defined on the convex cone spanned by differences of distributions $F-G$.

The local linearity assumption in the robustness setup can also be approached by mimicking classical requirements about the limiting distributions of regular estimators. Estimators $\hat{\theta}_{n}$ which include good competitors to the maximum likelihood estimator are usually required to satisfy the following asymptotic uniform distributional stability conditions (see for instance Le Cam 1986; Millar 1983):

$$
\sqrt{n}\left[\hat{\theta}_{n}-\left(\theta+\tau_{n} / \sqrt{n}\right)\right]_{P_{\theta+\tau_{n} / \sqrt{n}}^{\otimes n}} \rightarrow{ }_{w} M
$$

where $P^{\otimes n}$ stands for the product measure while the weak limit $M$ is a probability distribution (usually normal) independent of the choice of a bounded sequence $\tau_{n}$. These estimators are called regular and their asymptotic properties relative to asymptotically efficient estimators are explained by convolution theorems. We would then expect a "regular" functional to satisfy the following condition:

If the limit of $\sqrt{n}\left(G_{n}-F\right)$ in CvM norm exists and it is, say $g$, then the weak limit of $\sqrt{n}\left[T\left(F_{n}\right)-T(F)\right]_{G_{n}^{\otimes n}}$ exists, it is a shift of the distribution $M$, where

$$
\sqrt{n}\left[T\left(F_{n}\right)-T\left(G_{n}\right)\right]_{G_{n}^{\otimes n}} \rightarrow{ }_{w} M
$$

for $F_{n}$ the empirical distribution function and moreover the shifts are linear in $g$.

It was shown in Bednarski and Clarke (1998) that such regularization of the asymptotic properties of estimators, if applied to statistical functionals and extended to nonparametric neighborhoods of parametric models, also leads to the differentiability.

Theorem 3.1 Assume that in a CvM neighborhood of the model distribution $F$ the functional $T$ is bounded, $T\left(G_{1}\right)-T\left(G_{2}\right)=O\left(\left\|G_{1}-G_{2}\right\|\right)$ and the local linearity condition (2) holds. Then

- the functional $L$ is bounded and continuous for the $\mathrm{CvM}$ norm on the set of differences $G-F$

- it has the representation

$$
L(G-F)=\int \psi_{F} d(G-F)
$$

where $\psi_{F}(x)=L\left(I_{[x, \infty)}-F\right)$ is bounded, continuous and of finite variation

- the following expansion holds for the CvM norm

$$
\sqrt{n}\left[T\left(F_{n}\right)-T(F)\right]=\frac{1}{\sqrt{n}} \sum_{i=1}^{n} \psi_{F}\left(X_{i}\right)+o_{P}(1)
$$

where $F_{n}$ is the empirical distribution function based on the sample $X_{1}, \ldots, X_{n}$ from the distribution $G_{n}$. 
Proof The boundedness of $L$ follows from the local boundedness of $T$. For the continuity take $G_{n}$ converging to $G_{o}$ in CvM norm and define

$$
\tilde{G}_{n}=\left[1 / \sqrt{n} G_{n}+(1-1 / \sqrt{n}) F\right]
$$

and

$$
\tilde{G}_{n o}=\left[1 / \sqrt{n} G_{o}+(1-1 / \sqrt{n}) F\right] .
$$

Since $\tilde{G}_{n}-\tilde{G}_{n o}=\frac{1}{\sqrt{n}}\left(G_{n}-G_{o}\right)$ the sequence $\sqrt{n}\left|T\left(\tilde{G}_{n}\right)-T\left(\tilde{G}_{n o}\right)\right|$ converges to zero as $\mathrm{n}$ tends to infinity and

$$
\begin{array}{r}
\sqrt{n}\left[T\left(\tilde{G}_{n}\right)-T(F)-L\left(\tilde{G}_{n}-F\right)\right]=o\left(\left\|G_{n}-F\right\|\right) \\
\sqrt{n}\left[T\left(\tilde{G}_{n o}\right)-T(F)-L\left(\tilde{G}_{n o}-F\right)\right]=o\left(\left\|G_{n o}-F\right\|\right) .
\end{array}
$$

Taking the difference of the above two expressions we conclude the continuity.

For the representation of the functional define $x_{s}(t)=I_{[s, \infty)}-F(t)$ and put $\psi_{F}(s)=L\left(x_{s}\right)$. The continuity of $\psi_{F}$ follows from the continuity of $L$ and the fact that $s_{n} \rightarrow s_{O}$ implies $x_{s_{n}} \rightarrow x_{s_{o}}$ in CvM norm (always if $Q$ is continuous). The boundedness of $\psi_{F}$ results from the boundedness of the functional $L$. A standard argumentation for the Riemann-Stieltjes integration, using simple functions, leads then to the formula $L(G-F)=\int \psi_{F} d(G-F)$. Taking any strictly increasing sequence of numbers $\left\{s_{k}\right\}_{k=0, \ldots, n}$ and putting $Z_{n}(t)=\sum_{k=1}^{n}\left(x_{s_{k}}-x_{s_{k-1}}\right) \epsilon_{k}$, where $\epsilon=\operatorname{sgn}\left[\psi_{F}\left(s_{k}\right)-\psi_{F}\left(s_{k-1}\right)\right]$ gives

$$
\sum_{k=1}^{n}\left|\psi_{F}\left(s_{k}\right)-\psi_{F}\left(s_{k-1}\right)\right|=L\left(z_{n}\right) \leq\|L\|
$$

which yields the finite variation of $\psi_{F}$.

Finally since by Dvoretsky-Kiefer-Wolfowith inequality the random variables $\sqrt{n}\left\|F_{n}-G_{n}\right\|$ are bounded in probability and the sequence $\sqrt{n}\left\|G_{n}-F\right\|$ is also bounded we obtain the expansion from the approximate linearity condition and the validity of the representation.

In fact, by finite variation of the function $\psi_{F}$, we can deduce that the limiting distributions of $\sqrt{n}\left(T\left(F_{n}\right)-T(F)\right)$ under $G_{n}$ form the shifted normal family.

\section{The locally uniform Fréchet differentiability}

In as much as the boundedness and smoothness of the function $\psi_{F}$ respond to local robustness requirements, the usability of the expansion is also conditioned on its uniform validity-it should be possible to replace the distribution $F=F_{\theta}$ by other distributions from the parametric model in a local neighborhood of $F=F_{\theta}$. To stress the dependence of $\psi_{F}$ on model parameters it will be further more convenient to use 
the notation $\psi(\cdot, \theta)$. Theorem 3.1 in Bednarski et al. (1991) describes conditions that lead to the local uniformity of expansions. We adopt them here to possibly dependent sampling-conditions b3 and b4 below are expressed in terms of distances between the distribution functions rather than in terms of converging parameters as it is stated in the mentioned paper.

Let us denote by $\tau$ the parameter values in a neighborhood of $\theta$ and denote by $\psi(\cdot, \tau)$ the corresponding psi-functions defining the M-estimate. Finally put $K_{G}(\tau)=$ $\int \psi(x, \tau) d G(x)$. The following conditions lead to locally valid expansion:

b1 Functions $K_{G}(\tau)$ are continuous in $\tau$ in a neighborhood of $\theta$ for all $G$ in a CvM norm neighborhood of $F$

b2 $K_{F}(\theta)=0$ and $K_{F}(\tau)$ is differentiable at $\theta$ with nonsingular derivative $\dot{K}$.

b3 There is $c>0$ so that $\left|K_{G}(\theta)-K_{F}(\theta)\right| \leq c|| G-F \|$

b4 $\left|K_{G}(\tau)-K_{G}(\theta)-\left(K_{F}(\tau)-K_{F}(\theta)\right)\right|=o\left(\|G-F\|+\left\|F_{\tau}-F_{\theta}\right\|\right)$

Theorem 4.1 Under conditions (b1)-(b4) we have

$$
\left|T(G)-\tau-\dot{K}^{-1} K_{G}(\tau)\right|=o\left(\|G-F\|+\left\|F_{\tau}-F_{\theta}\right\|\right)
$$

Proof Since detailed considerations are given in the mentioned paper and the argumentation here is very similar, only the outline of the proof will be given.

From the differentiability of $K_{F}(\tau)$ and (b3) it follows, by a fixed point theorem, that we can find solutions $T(G)$ to the equation $K_{G}(\tau)=0$ so that $\sqrt{n}|T(G)-\theta|$ stays bounded if $\sqrt{n}\|G-F\|$ stays bounded. Now (b4) and again the differentiability of $K_{F}(\tau)$ gives

$$
K_{G}\left((T(G))-K_{G}(\tau)=\dot{K}(T(G)-\tau)+o\left(\|G-F\|+\left\|F_{\tau}-F_{\theta}\right\|\right)\right.
$$

\section{Testing unit root: simulation results}

This section is intended to show more practical merits of robust estimation based on Fréchet differentiable functionals which have the "highly reliable" expansions. Let us note that expansions are used firstly to establish limiting distributions of the estimators and in practical instances also to estimate their variability - therefore in our simulation study we mainly focus on distributional properties of estimates. There is a vast literature on central limit theorems for dependent variables that could be applied to the expansions. We give just a few references that seem most suitable for the models and methods studied in this paper. In Herrndorf (1984) and in Franck and Zakoian (2005) one can find results nested in the context of $\alpha$ mixing conditions. Another area of reference would correspond to so called $\psi$-weak dependence in Doukhan and Neumann (2008) and Dedecker et al. (2007).

The simulations were carried out for the process $Y_{t}=\rho Y_{t-1}+\epsilon_{t}$ with independent standard normal and Cauchy innovations. Estimation and testing were performed for series of length 200 and repeated 10,000 times. Contaminants are randomly "injected" 
Table 1 Fraction of acceptance of the unit root hypothesis at 0.05 significance level; for each parameter value the raws correspond to least squares, Tukey's biweight, adf and kpss method respectively

\begin{tabular}{lll}
\hline$\rho$ & $0 \%$ cont. & $2 \%$ cont. \\
\hline 1 & 0.91 & 0.55 \\
& 0.91 & 0.80 \\
& 0.96 & 0.92 \\
& 0.97 & 0.90 \\
0.95 & 0.07 & 0.56 \\
& 0.10 & 0.13 \\
& 0.85 & 0.91 \\
& 0.80 & 0.91 \\
& 0.00 & 0.14 \\
& 0.00 & 0.01 \\
& 0.61 & 0.78 \\
& 0.59 & 0.75 \\
\hline
\end{tabular}

five elements subseries with either $\rho=1.2$ or $\rho=0.5$, depending on the simulation case, $\rho<1$ and $\rho=1$ respectively. The chance of injection was 0.02 at each time unit. Two estimation methods were applied-least squares and a highly robust method based on Tukey's biweight score function with preliminary high breakdown point scale S-estimator. The unit root hypothesis was tested using the standardized estimates of the two methods and compared with augmented Dickey and Fuller test (Said and Dickey 1984) and Kwiatkowski et al. (1992) methods. The R language and tseries and MASS packages were used to do the simulations.

Table 1 shows high tendency to accept the unit root hypotheses by the general adf and kpss methods (unspecified order of the model) as compared to the least squares and the robust method under normal innovations for parameter values closed to 1 . Figures 1 and 2 show density histograms of the standardized estimator's values, normal density best fitting the data (continuous line) and the standard normal density (dashed lines). Standardization means here that the true parameter value is subtracted from the estimator and the difference is divided by an estimate of the standard deviation, based on the expansion in the robust estimator case. The shift between dashed and continuous lines show bias of the estimator-in Fig. 1 it results from nonstationarity of the series, in Fig. 2 it is the contamination that causes bias. The two curves virtually coincide in the noncontaminated case when $\rho=0.95$ and $\rho=0.9$ (graph not included). The overall better distributional behavior of the smooth estimator is clearly visible under contaminations. The study was also done for the Cauchy innovations. It shows similar overall testing and distributional behavior of the robust estimator. The least squares method leads there however to highly spread standardized estimate values.

Results of simulation discussed above indicate that use of smooth inferential robust procedures, in addition to the standard ones, may broaden our understanding of the nature of econometric series. Observed time series quite frequently behave in a way "close" to the unit root hypothesis: take for instance the series of exchange rates for the US dollar and the euro since July 2002 to July of 2007-the robust method rejects the unit root hypotheses there while the adf and kpss tests accept the hypothesis at 

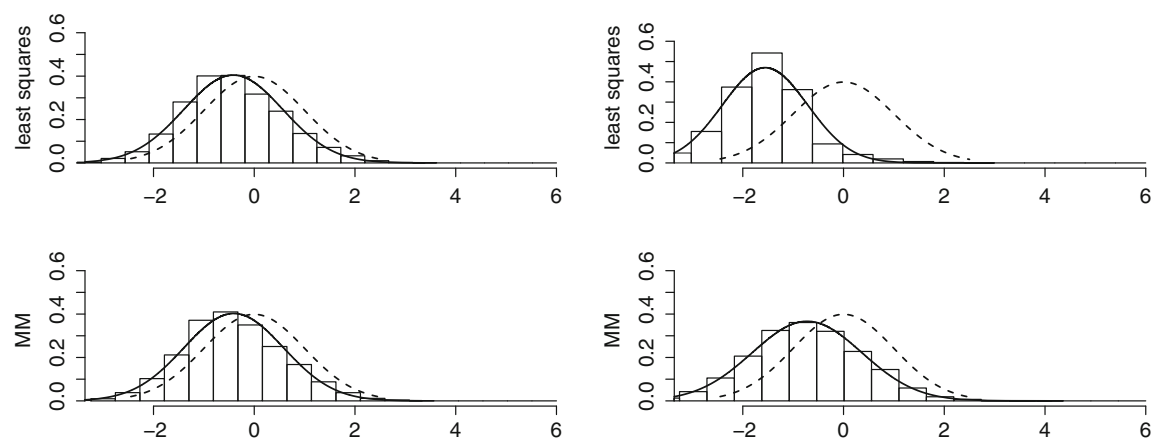

Fig. 1 Distributions of standardized estimates of $\rho=1$ in uncontaminated (left) and contaminated (right) random walk models. Upper graphs correspond to least squares, lower to robust estimation with Tukey's biweight score function
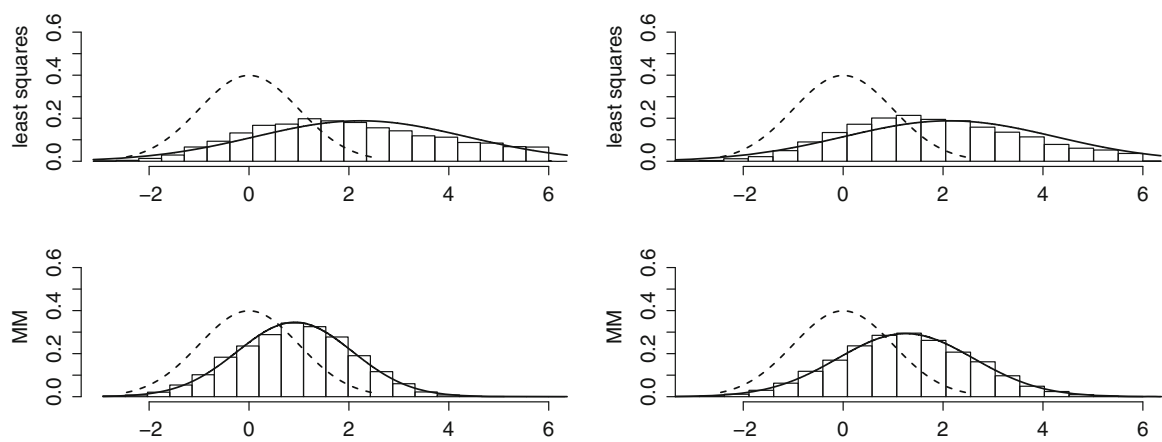

Fig. 2 Distributions of standardized estimates of $\rho=0.95$ (left) and $\rho=0.9$ (right) in the contaminated case. Upper graphs correspond to least squares, lower to robust estimation with Tukey's biweight score function

significance level 0.05. One may wonder about non-conclusiveness of statistical inference in such situations. Do we really want to distinguish, in our inference, the case $\rho=0.95$ from $\rho=1$ ? How important is the information about the unit root in explaining the economic phenomena? How can we usefully apply the information about the nearly unit root hypothesis? It would be good to have clear priorities here built in the context of econometric perspective. Some discussion of the above issues and an explanation of the extreme size distortion of the KPSS test are given in Cappuccio and Lubian (2010).

\section{Conclusions}

It is shown that Fréchet differentiability of a statistical functional with respect to the Cramer von Mises norm guarantees qualitative robustness of the corresponding estimator-its influence function has to be bounded and smooth. Even though a differentiable functional need not lead to robustly efficient estimator-its breakdown 
point may be close to zero while variance of the limiting distribution may be far from optimal-one can apply the general idea of the likelihood function truncation in conjunction with differentiability to derive reasonable robust procedures for statistical models where analytic complexity does not let us obtain optimal robust procedures (compare Bednarski 1993 and Bednarski and Mocarska 2006). It was demonstrated that locally differentiable functionals lead to locally uniform expansions and consequently to reliable asymptotic properties of the estimators not only at the model distributions but also at their small fully nonparametric vicinities. Those features, compulsory for statistical inference, are not inherited by any standard robustness criteria.

The differentiability notion may in particular be applied to facilitate asymptotic derivations in statistical inference for stochastic processes. We show that the the empirical distribution function is uniformly root- $n$ consistent for the Cramer von Mises norm under dependent observations satisfying relatively weak mixing conditions. The fact leads to asymptotic expansions for procedures used in statistical inference for stationary time series. The simulations show a high distributional stability and closeness to the normal law of estimates used to test the unit root hypotheses for time series under heavy contaminations and under symmetric innovations with heavy tails. It is also indicated that smooth robust procedures may be a good complement to standard procedures of testing the unit root hypotheses-because of their insensitivity to abrupt changes in asymptotic distributions of statistics while shifting from the stationary to the unit root case.

Open Access This article is distributed under the terms of the Creative Commons Attribution Noncommercial License which permits any noncommercial use, distribution, and reproduction in any medium, provided the original author(s) and source are credited.

\section{References}

Bednarski T, Clarke BR, Kolkiewicz W (1991) Statistical expansions and locally uniform Fréchet differentiability. J Austral Math Soc (Series A) 50:88-97

Bednarski T (1993) Robust estimation in the Cox regression model. Scand J Statist 20:213-225

Bednarski T, Clarke BR (1998) On locally uniform expansions for regular functionals. Discussiones Mathematicae. Algebra Stochastic Methods 18:155-165

Bednarski T, Mocarska E (2006) On robust model selection within the Cox model. Econ J 9:279-290

Cappuccio N, Lubian D (2010) The fragility of the KPSS stationarity test. Stat Methods Appl 19:237-253

Dedecker J, Doukhan P, Lang G, Len JR, Louhichi S, Prieur C (2007) Weak dependence: with examples and applications. Lecture Notes in Statistics, vol 190. Springer, Berlin

Doukhan P, Neumann MH (2008) The notion of $\psi$-weak dependence and its applications to bootstrapping time series. Probab Surveys 5:146-168

Dvoretzky A, Kiefer J, Wolfowitz J (1956) Asymptotic minimax character of the sample distribution function and of the classical multinomial estimator. Ann Statist 27:642-669

Franck Ch, Zakoian JM (2005) A central limit theorem for mixing triangular arrays of variables whose dependence is allowed to grow with the sample size. Econ Theory 21:1165-1171

Hampel FR, Ronchetti EM, Rousseeuw PJ, Stahel WA (1986) Robust statistics - the approach based on influence functions. Wiley, New York

Herrndorf N (1984) A functional central limit theorem for weakly dependent sequences of random variables. Ann Probab 12:141-153

Kwiatkowski D, Phillips PCB, Schmidt P, Shin Y (1992) Testing the null hypothesis of stationarity against the alternative of a unit root. J Econ 54:159-178 
Le Cam L (1986) Asymptotic methods in statistical decision theory. Springer-Verlag, New York

Mancini L, Ronchetti E, Trojani F (2005) Optimal conditionally unbiased bounded-influence inference in dynamic location and scale models. J Am Statist Assoc 100:628-641

Millar PW (1983) The minimax principle in asymptotic statistical theory. Springer Lect Notes Math 976: 75-265

Muler N, Pena D, Yohai VJ (2009) Robust estimation for ARMA models. Ann Statist 37:816-840

Phillips PCB (2004) Laws and limits in econometrics. Cowles foundation paper no. 1081

Rio E (2000) Inégalités de Hoeffding pour les fonctions lipschitziennes des suités dépendantes. C.R. Acad. Sci. Paris 1. 330, Série 1, pp 905-908, Probabilités

Said SE, Dickey DA (1984) Testing for unit roots in autoregressive-moving average models of unknown order. Biometrika 71:599-607 